\title{
ANÁLISE COMPARATIVA DE MÉTODOS DE ESTIMATIVA DE ÁREA FOLIAR EM CAFEEIRO(1)
}

\author{
JÚLIO EDUARDO TAVARES-JÚNIOR ${ }^{(2)}$; JOSÉ LAÉRCIO FAVARIN(3); DURVAL \\ DOURADO-NETO ${ }^{(3,6)}$; ALINE DE HOLANDA NUNES MAIA ${ }^{(2,4)}$; LUIZ CARLOS \\ FAZUOLI ${ }^{(5,6) ;}$ MARCOS SILVEIRA BERNARDES(3) $^{(3)}$
}

\begin{abstract}
RESUMO
Com o objetivo de avaliar a exatidão de três métodos empregados para a estimativa da área foliar em cafeeiro, realizou-se um experimento no Instituto Agronômico, em Campinas (SP). Coletaram-se, aleatoriamente, amostras de folhas da cultivar Mundo Novo IAC 388-17, em várias posições no dossel e em relação ao ramo plagiotrópico, a fim de estimar a área foliar, utilizando-se três métodos: BARROS, discos foliares e SIARCS 3.0, comparados com um método de referência (LI-COR). Os critérios utilizados na comparação dos métodos foram: a) coeficiente angular do modelo de regressão (b); b) erro relativo médio (ERM): média das diferenças entre os valores de área foliar obtidos pelo método alternativo e pelo método de referência, expressas como porcentagem dos valores obtidos no método de referência; c) coeficiente de determinação $\left(R^{2}\right)$, e d) padrão de dispersão dos erros. A incerteza, associada às estatísticas $R^{2}$ e ERM, foi calculada pelo procedimento de reamostragem denominado 'bootstrap'. O método SIARCS 3.0 foi o mais exato, considerado adequado para estimar a área foliar (valores absolutos) em substituição ao método LI-COR, ou para a calibração de métodos expeditos. Observa-se uma tendência de subestimar os valores de área foliar quando se utiliza o método proposto por BARros e de superestimá-los quando se utiliza o método de discos foliares.
\end{abstract}

Palavras-chave: Coffea arabica L., área foliar, estimativa, exatidão.

\section{ABSTRACT \\ COMPARATIVE ANALYSIS AMONG METHODS OF ESTIMATING COFFEE-TREE LEAF AREA}

With the purpose of evaluating the accuracy of three methods to estimate area of individual coffee leaves a field experiment was carried out at Agricultural Institute (IAC) at Campinas, São Paulo State, Brazil, where 50 coffee (cultivar Mundo Novo) leaves were randomized collected. At three methods of measurement (SIARCS 3.0, BARROS and leaves discs methods) were compared with the standard one (LI-COR) using simple regression analysis $(\mathrm{Yi}=\beta \mathrm{Xi}+\varepsilon \mathrm{i})$. The three methods were evaluated using the following criteria: a) slope of the linear regression model $(\beta)$; b) determination coefficient $\left(\mathrm{R}^{2}\right)$; $\mathrm{c}$ ) mean relative error (EMR), and d) dispersion pattern of residuals. The uncertainty associated to $\mathrm{R}^{2}$ and EMR was assessed using the bootstrap procedure. The SIARCS 3.0 method was the most accurate one, being useful to estimate leaf area (absolute values), or to calibrate expeditious methods, instead the LI-COR method. The BARROS and the leaf disc methods showed a tendency of underestimating and overestimating the leaf area values, respectively.

Key words: Coffea arabica L., leaf area, methodology, accuracy.

( $\left.{ }^{1}\right)$ Recebido para publicação em 27 de novembro de 2001 e aceito em 9 de maio de 2002.

$\left(^{2}\right)$ Pós-graduação em Agronomia, área de Fitotecnia, Escola Superior de Agricultura "Luiz de Queiroz", Universidade de São Paulo (ESALQ/USP), Caixa Postal 9, 13418-970 Piracicaba (SP). E-mail: jetavare@esalq.usp.br

(3) Departamento de Produção Vegetal, ESALQ/USP. E-mail: jlfavari@esalq.usp.br

$\left({ }^{4}\right)$ Embrapa Meio Ambiente, Caixa Postal 69, 13820-000 Jaguariúna (SP). E-mail: amaia@cnpma.embrapa.br

$\left(^{5}\right)$ Centro de Análise e Pesquisa do Agronegócio do Café, Instituto Agronômico (IAC), Caixa Postal 28, $13001-970$ Campinas (SP). E-mail: ritacc@cec.iac.br

$\left(^{6}\right)$ Com bolsa de produtividade científica do CNPq. 
A determinação da área foliar do cafeeiro é fundamental para estudar aspectos fisiológicos que envolvam análise de crescimento e fotossíntese, transpiração, bem como quantificar os danos causados por pragas e doenças foliares. No aspecto prático, pode-se estimar a perda de água pelo cafeeiro (FAVARIN et al., 2001), contribuindo, assim, para maior eficiência econômica na utilização da água, uma vez que se trata de recurso escasso, por meio do manejo adequado da irrigação. A folha é o principal órgão no processo transpiratório, responsável pelas trocas gasosas entre a planta e o ambiente (Pereira et al., 1997), razão pela qual o conhecimento da superfície foliar é de grande utilidade para a avaliação de outras técnicas culturais como poda, adubação, densidade de plantio e aplicação de defensivos.

No trabalho realizado por HUERTA (1962), com o objetivo de comparar a eficiência de métodos de laboratório e de campo para estimar a área foliar em cafeeiro, dentre os quais o método fotográfico, massa de discos foliares, método gráfico de comparação de superfícies e método de dimensões foliares, concluiuse que não há diferença entre os resultados obtidos e seus respectivos métodos.

Com o intuito de estimar a área foliar, BARros et al. (1973) utilizaram como padrão o método gravimétrico descrito por Kemp (1960) e Huerta e Alvim (1962), correlacionando, por regressão linear, os valores encontrados àqueles obtidos com as dimensões dos retângulos circunscritos aos limbos foliares. Com base nos resultados, os autores apresentaram a equação $A=0,667$.C.L (C: maior comprimento e L: maior largura) para estimar a superfície foliar de cafeeiro.

Dentre os métodos conhecidos que auxiliam a determinação da área foliar em plantas de café (HUERTA, 1962; AWATRAMANI e GOPALAKRISHNA, 1965; BARROs et al., 1973; GoMIDE et al., 1977), merece destaque, para outras culturas, o procedimento que relaciona a área foliar com as dimensões lineares da folha (PEREIRA, 1987), devido à rapidez na obtenção de dados, quando se utiliza amostra de folhas, e por não ser, necessariamente, destrutivo (PEDRO-JúnIOR et al., 1986).

No intuito de obter dados confiáveis de maneira rápida e eficiente, estudaram-se métodos que envolvem técnicas de digitalização de imagens, como o SIARCS 3.0 (Sistema Integrado para Análise de Raízes e Cobertura do solo), uma versão para Windows do software desenvolvido como parte do sistema SIARCS (CRESTANA et al., 1994; Jorge e CRestana, 1996).

O presente trabalho teve por objetivo avaliar a exatidão de três métodos expeditos (BARRos et al.,
1973, discos foliares e SIARCS 3.0) utilizados para estimar a área foliar de cafeeiros, assumindo-se que os resultados obtidos com o integrador de área foliar LICOR (método de referência) são exatos, utilizando uma cultivar amplamente difundida nas regiões cafeeiras.

\section{Material e Métodos}

O experimento foi realizado no Núcleo Experimental de Campinas, Instituto Agronômico, Campinas, Estado de São Paulo. Com base em amostras de 50 folhas coletadas aleatoriamente, em várias posições no dossel e em relação ao ramo plagiotrópico, de plantas de café da cultivar Mundo Novo IAC 38817, estimou-se a área foliar, utilizando-se três métodos: BARROS et al., 1973; discos foliares e SIARCS 3.0.

No método de referência (LI-COR), utiliza-se um aparelho digital integrador de área, cuja teoria operacional fundamenta-se no princípio de células de grade de área conhecida (LI-COR 3100, 1996).

O SIARCS 3.0 (Sistema Integrado para Análises de Raízes e Cobertura do Solo), desenvolvido pela EMBRAPA/CNPDIA, cujo método é descrito por JoRGE e CRESTANA (1996), foi utilizado para verificar sua exatidão a fim de substituir o método admitido como referência (LI-COR), em vista do menor custo para sua aquisição e manutenção. Nesse método, as imagens digitalizadas de folhas são obtidas por um scanner de mesa e enviadas para um arquivo de computador para serem processadas pelo software SIARCS (versão 3.0). A área foliar é avaliada utilizando um algoritmo, integrando a superfície digitalizada por meio da técnica de medida e classificação de objetos em imagens digitais.

O método de discos foliares consiste na retirada de discos foliares de área conhecida em quatro posições do limbo foliar de um conjunto de folhas, distribuídas simetricamente, evitando-se a amostragem da nervura central, conforme estudos de Huerta (1962) e Gomide et al. (1977). Em seguida, os discos foliares, de área conhecida, foram colocados em estufa com circulação de ar a $70^{\circ} \mathrm{C}$ durante 36 horas para a obtenção da matéria seca. Utilizou-se procedimento semelhante para a secagem das folhas das quais foram retirados os discos foliares, cuja massa resultou do somatório das folhas com a dos discos foliares, obtendo-se a matéria seca total das folhas.

No método de BARRos et al. (1973), a estimativa da área foliar baseia-se nas dimensões do retângulo circunscrito aos limbos foliares, ajustados pela equação $Y=0,667 . X$, em que: $Y$ representa a área foliar e $X$, a área do retângulo circunscrito ao limbo 
foliar, obtida pelo produto entre o maior comprimento e a maior largura da folha.

Para verificar a exatidão dos métodos foram, inicialmente, ajustados modelos de regressão linear simples sem interceptação, relacionando-se os valores de área foliar obtidos pelo método de referência (LI-COR) com os valores de cada um dos demais métodos avaliados, por meio de:

$$
Y_{i}=\beta X_{i}+\varepsilon_{i}
$$

em que: $Y_{i}$ representa a área da i-ésima folha obtida pelo método alternativo avaliado; $\beta$, o coeficiente angular; $X_{i}$ corresponde à área da i-ésima folha obtida, utilizando o método LI-COR, e $\varepsilon_{\mathrm{i}^{\prime}}$ ao erro aleatório associado a cada observação.

O parâmetro $\beta$ foi estimado pelo método de quadrados mínimos. Para cada método alternativo, testou-se a hipótese $\beta=1$, que corresponde à equivalência entre as medidas de área foliar, utilizando o método alternativo ou o método de referência.

Sob tal hipótese de equivalência, ou seja, modelo de regressão linear com interceptação nula e coeficiente linear unitário, calculou-se o coeficiente de determinação $\left(R^{2}\right)$, relação entre as somas de quadrados de desvios dos valores de área foliar obtidos nos métodos LI-COR e cada um dos métodos avaliados.

Para cada método alternativo, estimaram-se o erro relativo médio (ERM), média do valor das diferenças (em módulo) entre os valores de área foliar obtidos pelo método alternativo e pelo método de referência, expressas como porcentagem dos valores obtidos no método de referência. Para avaliar o padrão de dispersão dos desvios, elaboraram-se gráficos dos desvios observados para cada método alternativo, em função dos valores de área foliar obtidos pelo método SIARCS. Esses gráficos permitem detectar tendências de sub- ou superestimação, que não podem ser captadas pelas estatísticas ERM ou $\mathrm{R}^{2}$
Os três métodos avaliados foram comparados com o método de referência, com base nos seguintes critérios: a) coeficiente angular do modelo de regressão sem interceptação $(\beta) ; b)$ erro relativo médio (ERM); c) coeficiente de determinação $\left(R^{2}\right)$ e d) padrão de dispersão dos erros.

A incerteza, associada às estatísticas ERM e $\mathrm{R}^{2}$, foi estimada pelo método de reamostragem denominado 'bootstrap' (EFrON e TiBSHIRANI, 1993). Foram simulados valores de área foliar correspondentes a cada um dos métodos para um conjunto de 5.000 amostras de 49 folhas. Utilizando os 5.000 valores de ERM e $R^{2}$ correspondentes a cada grupo de 49 folhas, avaliaram-se as estimativas 'bootstrap' do ERM e $\mathrm{R}^{2}$ e seus intervalos de confiança.

\section{Resultados e Discussão}

Os resultados referentes ao ajuste dos modelos de regressão sem interceptação para cada método avaliado são apresentados no quadro 1 . Os gráficos dos modelos de calibração, estabelecendo-se a relação entre as medidas obtidas pelo método LI-COR (referência) com os demais métodos e o padrão de dispersão dos desvios observados em cada um dos métodos alternativos, são apresentados nas figuras 1 e 2 respectivamente.

A concordância entre o método de referência (LICOR) e os alternativos é indicada pela estimativa do coeficiente angular do modelo de regressão sem interceptação $(\beta)$, erro relativo médio (ERM), coeficiente de determinação $\left(R^{2}\right)$ e padrão de dispersão dos erros. Valores de $b$ próximos da unidade, $\mathrm{R}^{2}$ perto de $100 \%$, EMR mínimo e padrão de dispersão dos erros completamente aleatório são desejáveis.

Considerando-se que as estatísticas $\mathrm{R}^{2}$ e EMR possuem uma incerteza a elas associada, decorrente do fato de terem sido estimadas por intermédio de uma amostra de folhas, foram simulados valores de $\mathrm{R}^{2}$ e EMR que poderiam ser obtidos se fossem

Quadro 1. Estimativas dos coeficientes angulares dos modelos de regressão linear sem intercepto com seus respectivos erros-padrão e intervalos de confiança de 95\% (limites inferior e superior)

\begin{tabular}{|c|c|c|c|c|}
\hline \multirow{2}{*}{ Método } & \multicolumn{2}{|c|}{ Coeficiente angular $(\beta)$} & \multirow[b]{2}{*}{ Limite inferior } & \multirow[b]{2}{*}{ Limite superior } \\
\hline & Estimativa & Erro-padrão & & \\
\hline BARROs et al. (1973) & $0,9476^{\mathrm{ns}}$ & 0,0070 & 0,8586 & 1,0367 \\
\hline Discos foliares & $1,0968^{*}$ & 0,0068 & 1,0098 & 1,1839 \\
\hline SIARCS 3.0 & $1,0032^{\mathrm{ns}}$ & 0,0043 & 0,9488 & 1,0576 \\
\hline
\end{tabular}

ns: Hipótese $\beta=1$, não rejeitada em nível de significância de 5\%. : Hipótese $\beta=1$, rejeitada em nível de significância de $5 \%$. 

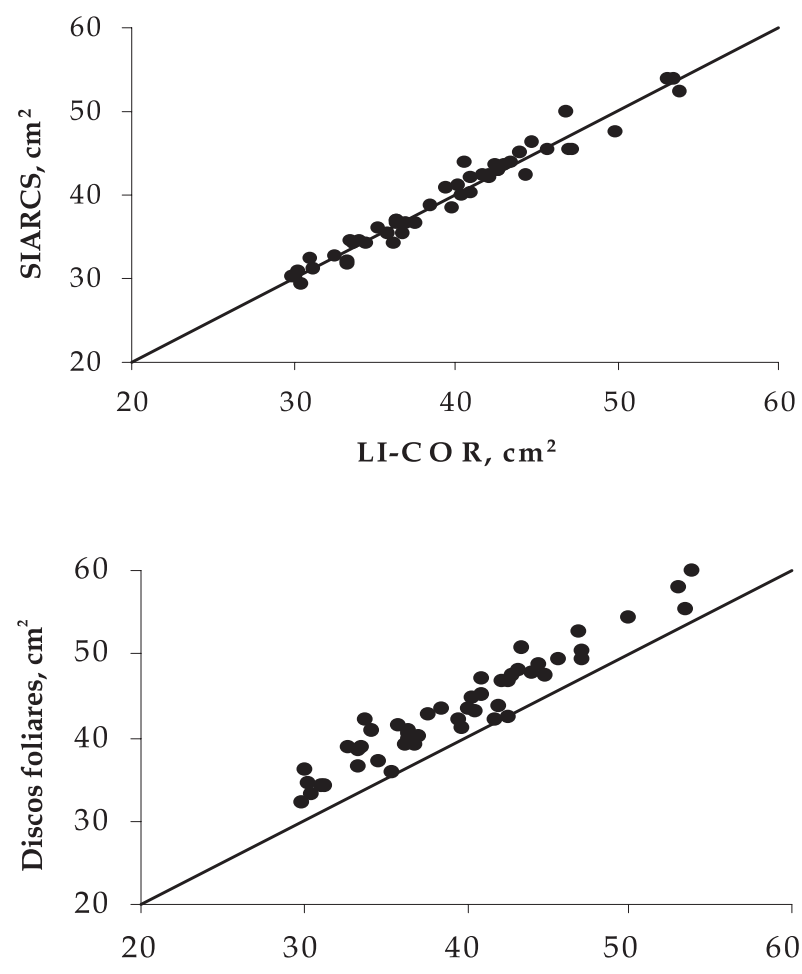

LI-C O R, $\mathrm{cm}^{2}$

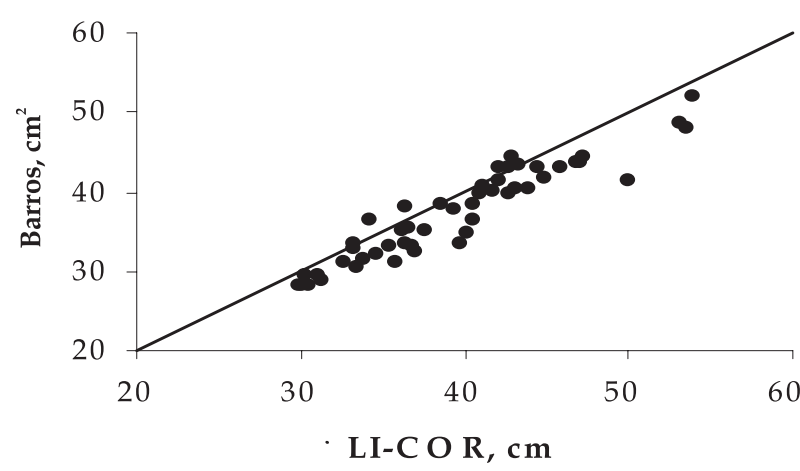

Figura 1. Modelos de calibração para a estimativa de área foliar, comparando-se o método referência (X, LI-COR) com os demais métodos (Y): SIARCS, discos foliares e BArRos et al. (1973). utilizadas outras amostras de folhas, adotando-se o método de reamostragem denominado 'bootstrap'. As estimativas do erro médio relativo (EMR) e do coeficiente de determinação $\left(R^{2}\right)$ e seus respectivos limites superior e inferior do intervalo de confiança de $95 \%$ constam do quadro 2.

Quanto menor o valor de EMR, maior é a exatidão da estimativa da variável (área foliar). Com relação a esse critério, observou-se que os métodos SIARCS e BARRos et al. (1973), nessa ordem, foram superiores ao método de discos foliares (Quadro 2 e Figura 2).

De acordo com os resultados apresentados no quadro 1, pode-se afirmar que o método SIARCS é o que se aproxima mais do método de referência (LICOR), podendo substituí-lo em estudos de calibração de métodos expeditos. A adoção do método SIARCS apresenta vantagem econômica devido ao menor custo de obtenção e operacional do software (SIARCS, versão 3.0) comparado ao custo de aquisição e manutenção do equipamento de marca LI-COR (modelo 3100).

Dentre os métodos expeditos, o SIARCS apresentou maior coeficiente de determinação $\left(R^{2}\right)$ e exatidão superior para a estimativa da área foliar, quando comparado aos métodos de discos foliares e BARROS et al. (1973) - Quadro 2.

Nota-se em trabalhos comparativos que há restrições aos métodos de discos foliares e BARROS et al. (1973), pois esses apresentaram baixa precisão (Figura 2).

Para estudos que visam ao conhecimento do valor absoluto da área foliar, o método SIARCS é mais exato em relação aos demais, uma vez que o modelo de calibração ajustado apresenta coeficiente angular próximo a um (Quadro 1), $R^{2}$ elevado e EMR baixo (Quadro 2) e padrão aleatório de dispersão de erros (Figura 2).

Os resultados do presente trabalho não concordam com os de Gomide et al. (1977), pois esses autores, ao avaliar a área foliar de 'Mundo Novo' e 'Catuaí', observaram que o método Barros et al.

Quadro 2. Estimativas "bootstrap" do erro relativo médio (ERM) e do coeficiente de determinação ( $\left.\mathrm{R}^{2}\right)$, com respectivos intervalos de confiança de $95 \%$ (limites inferior e superior) para os três métodos de determinação de área foliar de cafeeiro

\begin{tabular}{|c|c|c|c|c|c|c|}
\hline \multirow[t]{2}{*}{ Método } & \multicolumn{3}{|c|}{ Erro relativo médio } & \multicolumn{3}{|c|}{ Coeficiente de determinação } \\
\hline & Estimativa & $\begin{array}{l}\text { Limite } \\
\text { inferior }\end{array}$ & $\begin{array}{c}\text { Limite } \\
\text { superior }\end{array}$ & Estimativa & $\begin{array}{l}\text { Limite } \\
\text { inferior }\end{array}$ & $\begin{array}{c}\text { Limite } \\
\text { superior }\end{array}$ \\
\hline & & - \% & & & - \% & \\
\hline BARROS et al. (1973) & 5,81 & 4,92 & 6,74 & 92,40 & 89,57 & 94,73 \\
\hline Discos foliares & 10,23 & 9,16 & 11,32 & 92,44 & 89,73 & 94,73 \\
\hline SIARCS 3.0 & 2,49 & 2,06 & 2,94 & 95,21 & 96,48 & 97,55 \\
\hline
\end{tabular}



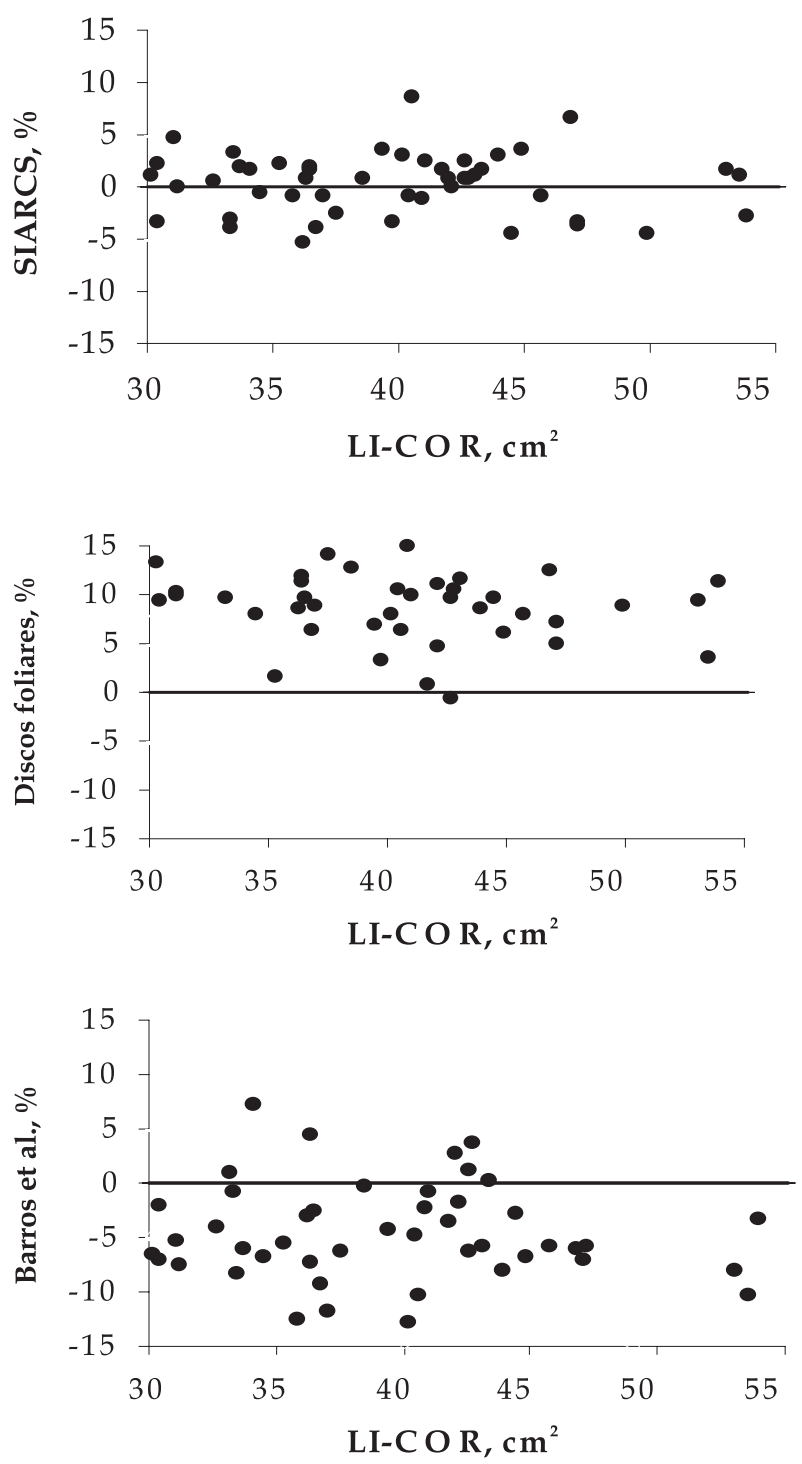

Figura 2. Dispersão dos erros relativos dos métodos SIARCS, discos foliares e BARROs et al. (1973).

(1973) apresentou maior exatidão do que o de discos foliares e o método fotográfico, este admitido como padrão. HUERTA (1962), empregando vários métodos (laboratoriais e de campo) para estimar a área foliar em cafeeiro, dentre os quais o de discos foliares e o de relação entre a área foliar e as dimensões lineares da folha, observou que não houve diferença nem quanto à precisão, nem quanto à exatidão.

\section{Referências Bibliográficas}

AWATRAMANI, D.A.; GOPALAKRISHNA, H.K. Measurement of leaf area (Coffea arabica L.). Indian Coffee, Bangalore, v.29, n.1, p.61-64, 1965.
BARROS, R.S.; MAESTRI, M.; VIEIRA, M.; BRAGAFILHO, L.J. Determinação de área de folhas do café (Coffea arabica L.cv. 'Bourbon Amarelo'). Revista Ceres, Viçosa, v.20, n.107, p.44-52, 1973.

CRESTANA, S.; GUIMARÃES, M.F.; JORGE, L.A.C.; RALISCH, R.; TOZZI, C.L.; TORRENETO, A.; VAZ, C.M.P. Avaliação da distribuição de raízes no solo auxiliada por processamento de imagens digitais. Revista Brasileira de Ciência do Solo, Campinas,v.18, n.3, p.365-371, 1994.

EFRON, B.; TIBSHIRANI, R.J. An introduction to the bootstrap. New York: Chapman \& Hall, 1993. 436p.

FAVARIN, J.L.; VILLA NOVA, N.A.; ANGELOCCI, L.R.; DOURADO-NETO, D.; BERNARDES, M.S. Estimativa do consumo hídrico do cafeeiro em função de parâmetros climatológicos. In: SIMPÓSIO DE PESQUISA DOS CAFÉS DO BRASIL, 2., 2001, Vitória. Resumos... Brasília: Embrapa Café, 2001. p.47-48.

GOMIDE, M.B.; LEMOS, O.V.; TOURINO, D.; CARVALHO, M.M.; CARVALHO, J.G.; DUARTE, C.S. Comparação entre métodos de determinação de área foliar em cafeeiros Mundo Novo e Catuaí. Ciência Prática, Lavras, v.1, n.2, p.118-123, 1977.

HUERTA, S.A. Comparación de métodos de laboratorio y de campo para medir el area del cafeto. Cenicafé, Chinchina, v.13, n.1, p.33-42, 1962.

HUERTA, S.A.; ALVIM, P.T. Indice de area foliar y su influencia en la capacidad fotosintetica del cafeto. Cenicafé, Chinchina, v.13, n.2, p.75-84, 1962.

JORGE, L.A.C.; CRESTANA, S. SIARCS 3.0: novo aplicativo para análise de imagens digitais aplicado a ciência do solo. In: CONGRESSO LATINO AMERICANO DE CIÊNCIA DO SOLO, 13., 1996, Águas de Lindóia, $C D-R O M$. Campinas: Sociedade Brasileira de Ciência do Solo, 1996. 5p.

KEMP, C.D. Methods of estimating the leaf area of grasses from linear measurements. Annals of Botany, Oxford, v.24, n.96, p.491-499, 1960.

LI-COR. LI 3100 area meter instruction manual. Lincoln: LICOR, 1996. 34p.

PEDRO-JÚNIOR, M.J.; RIBEIRO, I.J.; MARTINS, F.P. Determinação da área foliar em videira cultivar Niágara Rosada. Bragantia, Campinas, v.45, n.1, p.199-204, 1986.

PEREIRA, A.R. Estimativa da área foliar em milharal. Bragantia, Campinas, v.46, n.1, p.147-150, 1987.

PEREIRA, A.R.; VILLA NOVA, N.A.; SEDIYAMA, R. Evapotranspiração. Piracicaba: FEALQ/ESALQ/USP, 1997. 70p. 